\title{
Heart-on-a-Chip Model with Integrated Extra- and Intracellular Bioelectronics for Monitoring Cardiac Electrophysiology under Acute Hypoxia
}

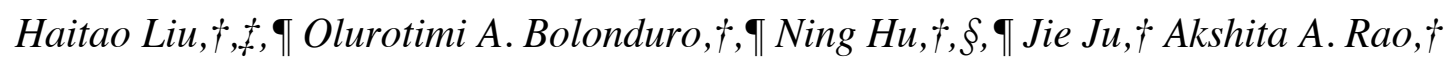

Breanna M. Duffy, $\dagger$ Zhaohui Huang,, Lauren D. Black,,++ Brian P. Timko*, $\dagger$

$\dagger$ Department of Biomedical Engineering, Tufts University, Medford, Massachusetts

02155, United States

+ School of Materials Science and Technology, Beijing Key Laboratory of Materials Utilization of Nonmetallic Minerals and Solid Wastes, National Laboratory of Mineral Materials, China University of Geosciences, Beijing 100083, PR China

$\S$ State Key Laboratory of Optoelectronic Materials and Technologies, School of Electronics and Information Technology, Guangdong Province Key Laboratory of Display Material and Technology, The First Affiliated Hospital of Sun Yat-Sen University, Sun Yat-Sen University, Guangzhou 510275, PR China

+ Department of Cell, Molecular \& Developmental Biology, School of Graduate Biomedical Sciences, Tufts University, Boston, Massachusetts 02111, United States 



\section{Materials and Methods}

Chemicals and reagents. Four-inch silicon wafers were purchased from University Wafer. TFG nickel etchant was from Transene Company, Inc. Polydimethylsiloxane (PDMS) was formed using a two-part kit (Sylgard 184) from Dow Corning. Chloroplatinic acid and lead acetate were purchased from Sigma-Aldrich. All reagents used for HL-1 cell culture were purchased from Sigma-Aldrich: gelatin, fibronectin, Claycomb medium, fetal bovine serum, norepinephrine, L-glutamine, penicillin, and streptomycin.

Planar MEA fabrication. Electrode patterns were designed with AutoCAD software (Autodesk Inc., USA). Planar MEAs (Fig. S1) were fabricated by standard photolithography followed by metallization with $10 \mathrm{~nm} \mathrm{Cr} / 40 \mathrm{~nm} \mathrm{Au}$. The conductive lines were passivated by $2 \mu \mathrm{m}$ thick SU-8 photoresist (Microchem SU-8 2002). The microelectrodes (30 $\mu \mathrm{m}$ diameter) were electroplated with platinum black using aqueous chloroplatinic acid $(1 \% \mathrm{w} / \mathrm{v})$ and lead acetate $(0.01 \% \mathrm{w} / \mathrm{v})$ at $-2 \mathrm{~V}$ for $15 \mathrm{~s}$ using a Gamry $600+$ electrochemical workstation (two electrode setup, Pt counter electrode). ${ }^{1}$

Vertical nanopillar fabrication. Gold pads and interconnects were fabricated by photolithography as described above. $\mathrm{A} 200 \mathrm{~nm} \mathrm{SiO} 2$ layer, followed by a $5 \mathrm{~nm}$ nickel layer, were then deposited by RF sputter. Vertical nanowire electrodes were defined on the gold pads by Focused Ion Beam (Tescan Lyra3, FEG-SEM/FIB, Czech). ${ }^{2}$ The nickel layer was then etched by TFG nickel etchant (Transene). Scanning electron microscope (SEM) images of the nanopillar electrodes were obtained using the Tescan Lyra3. A schematic illustration is shown in Scheme S1. 
Microfluidic channel. A microfluidic channel template was laser cut from poly(methyl methacrylate $($ PMMA) plates $(16 \mathrm{~mm} \times 3 \mathrm{~mm} \times 1.58 \mathrm{~mm}$; volume $=76 \mu \mathrm{l})$. Microfluidic channels were formed in polydimethylsiloxane (PDMS) using the template as a mold; base and curing agent (Sylgard 184) were pre-mixed in a $1: 10$ ratio, then cured at $80{ }^{\circ} \mathrm{C}$ for at least $2 \mathrm{~h}$ as per manufacturer instructions. The PDMS channels were cut to the appropriate size, treated with oxygen plasma to expose silanol groups, then immediately adhered to chips (Figure S1). Fluid was delivered to and from the channel through polyethylene / poly(vinyl chloride) (PE/PVC) tubes (Instech Laboratories, Inc.) using a programmable syringe pump (Harvard Apparatus PHD ULTRA) as shown in Scheme S2.

Electronic interface. Chips were mounted onto a custom printed circuit board (PCB). Electrical connections between the chip and PCB were achieved using silver epoxy (Chemtronics CW2400) coated with a layer of insulating epoxy (Grainger 5A462). External electronics were connected to the PCB via a 10-pin surface-mounted connector (1.27 $\mathrm{mm}$ or $2.54 \mathrm{~mm}$ pitch).

Cell culture. HL-1 cells (Louisiana State University Health Science Center, New Orleans, LA, USA; Passage 4-9) were expanded in Claycomb media with 10\% FBS, 100 $\mathrm{U} / \mathrm{ml}$ penicillin and streptomycin, $2 \mathrm{mM}$ L-glutamine and $0.1 \mathrm{mM}$ norepinephrine. The chips were coated with fibronectin by injecting $5 \mu \mathrm{g} / \mathrm{ml}$ fibronectin in $0.02 \%$ gelatin solution into the microchannel at $37^{\circ} \mathrm{C}$ for a least $1 \mathrm{~h}$. After removal of the gelatin fibronectin solution, a suspension of HL-1 cells $\left(\sim 2 \times 10^{5}\right.$ cells $\left./ \mathrm{ml}\right)$ was then introduced into the channel in the channel and left static for at least $3 \mathrm{~h}$ for seeding. Culture media was 
then flowed through the channel at $40 \mu \mathrm{L} \mathrm{h}^{-1}$ and this flow was maintained for the duration of culture, until induction of hypoxia. The chips were maintained in a standard incubator at $37{ }^{\circ} \mathrm{C}, 5 \% \mathrm{CO}_{2}$. Spontaneous beating was observed after 3-4 days.

Immunohistochemistry. Cardiac monolayers were fixed in 4\% paraformaldehyde in phosphate buffered saline (PBS, $\mathrm{pH} 7.4$ ) for 10 minutes, washed $3 \mathrm{x}$ in ice-cold PBS, and then permeabilized with PBS containing $0.25 \%$ Triton $\mathrm{X}-100$ for 10 minutes at room temperature. Following 3 buffer washes, the samples were incubated in 1\% BSA in PBST (PBS $+0.1 \%$ Tween 20) for 30 minutes. Samples were incubated for 1 hour with the appropriate primary antibodies diluted in blocking solution: mouse anti- $\alpha$-actinin (A7811, Sigma Aldrich, St. Louis, MO, 1:500), rabbit anti connexin-43 (ab11370, Abcam Cambridge, MA, 1:500), and rabbit anti-HIF-1 $\alpha$ (ab179483, Abcam Cambridge, MA, 1:500). After incubation, the samples were washed and incubated for 1 hour with a 1:500 dilution of secondary antibodies in blocking solution: Alexa Fluor 488 goat anti-mouse (A11001, ThermoFisher, Waltham, MA) and Alexa Fluor 568 donkey-anti-rabbit (A10042, ThermoFisher, Waltham, MA). For nuclear labeling the cells were incubated for $1 \mathrm{~min}$ with DAPI (Abcam, Cambridge, MA) and washed. Imaging was performed with a Leica TCS SP8 spectral confocal microscope.

HIF-1 $\alpha$ quantification. Confocal images of cardiac monolayers, which had been fluorescently marked for DAPI and HIF-1 $\alpha$, were post-processed in the NIH ImageJ/FIJI package. Briefly, images were subjected to watershed, despeckle, and remove outliers features of FIJI to prepare for quantification of HIF-1 $\alpha$ fluorescence within the nuclei of 
the cardiac monolayer. The region of interest was defined with the DAPI layer, and the outline of the nuclei superimposed onto the DAPI and HIF-1 $\alpha$ layers to measure fluorescence intensity. The ratio of HIF-1 $\alpha$ and DAPI fluorescence was calculated and averaged to quantify HIF-1 $\alpha$ expression in response to hypoxic media.

Hypoxic media preparation. Hypoxic media was prepared by continuously bubbling the cell culture media with ultrapure nitrogen gas saturated with water, as shown in Scheme S1. The oxygen concentration was monitored by an oxygen sensor (Atlas Scientific) interfaced with an analog-to-digital converter (Axon ${ }^{\mathrm{TM}}$ Digidata 1550B). Normoxic and hypoxic media were switched as described in Figs. 2a and S5a. Immediately upon switching, the flow rate was increased from $40 \mu \mathrm{l} / \mathrm{h}$ to $25 \mu \mathrm{l} / \mathrm{s}$ (time constant $\tau=$ volume / flow rate $=3 \mathrm{~s}$ ) for $10 \mathrm{~s}$ to completely flush the channel.

Movie recording. Supplementary Movies were recorded using an inverted microscope (Lecia Microsystems DMi1).

Impedance measurements. Electrical impedance spectroscopy of electrodes was measured in Claycomb culture medium using an electrochemical workstation (Gamry Reference 600+, Gamry Instruments, Inc., USA) using a three electrode setup with $\mathrm{Ag} / \mathrm{AgCl}$ reference electrode and on-chip Au pad $(2 \times 3 \mathrm{~mm})$ counter electrode. We scanned between 10 and $10^{6} \mathrm{~Hz}$ with a perturbative potential of $20 \mathrm{mV}$.

MEA measurements. Signals were acquired using a 16-channel Multielectrode Array (MEA) system (USB-ME16-FAI, Multichannel Systems GmbH, Germany). The signals were amplified by $1000 \mathrm{x}$ at the headstage preamplifier, then sampled at $25 \mathrm{kHz}$. Signals 
were digitally filtered in MATLAB using a $60 \mathrm{~Hz}$ stopband and/or $80-2000 \mathrm{~Hz}$ bandpass filter.

Electroporation. Cells were electroporated with 60-100 biphasic square pulses (3 Vpp, $200 \mu$ s period, $50 \%$ duty) applied to the nanopillar electrodes applied using a function generator (Rigol DG1032). We found that the same cell could be electroporated at least three times, on three separate days (e.g. days 3, 4 and 5 days in vitro; data not shown). In the case of Figure 4, each group consisted of N=14 different cells measured across 4 different chips. Each time point represents a unique population of devices and cells. In the case of Figure 5, we applied the same biphasic square pulse sequence to 8 devices simultaneously. We used the same chip for normoxia and $2 \mathrm{~h}$ hypoxia time points but measured different devices and cells.

Isochronal maps. Time delays and isochronal maps (Figure 2,5) were calculated in MATLAB (MathWorks Inc., Natick, MA) by cross-correlation analysis ${ }^{3,4}$ on a $2 \mathrm{~s}$ segment of each trace. A matrix of vectors representing the gradient of the isochrones was calculated using the quiver function. The velocity corresponding to each vector was calculated by taking its inverse and multiplying by the appropriate scaling factor. These velocities were then averaged to find the average propagation speed.

Statistical analysis. Statistical data are presented as means \pm s.d. Groups were compared using Welch's t-test or two-tailed t-test; $\mathrm{P}<0.05$ was considered significant. All calculations were performed in GraphPad Prism. 

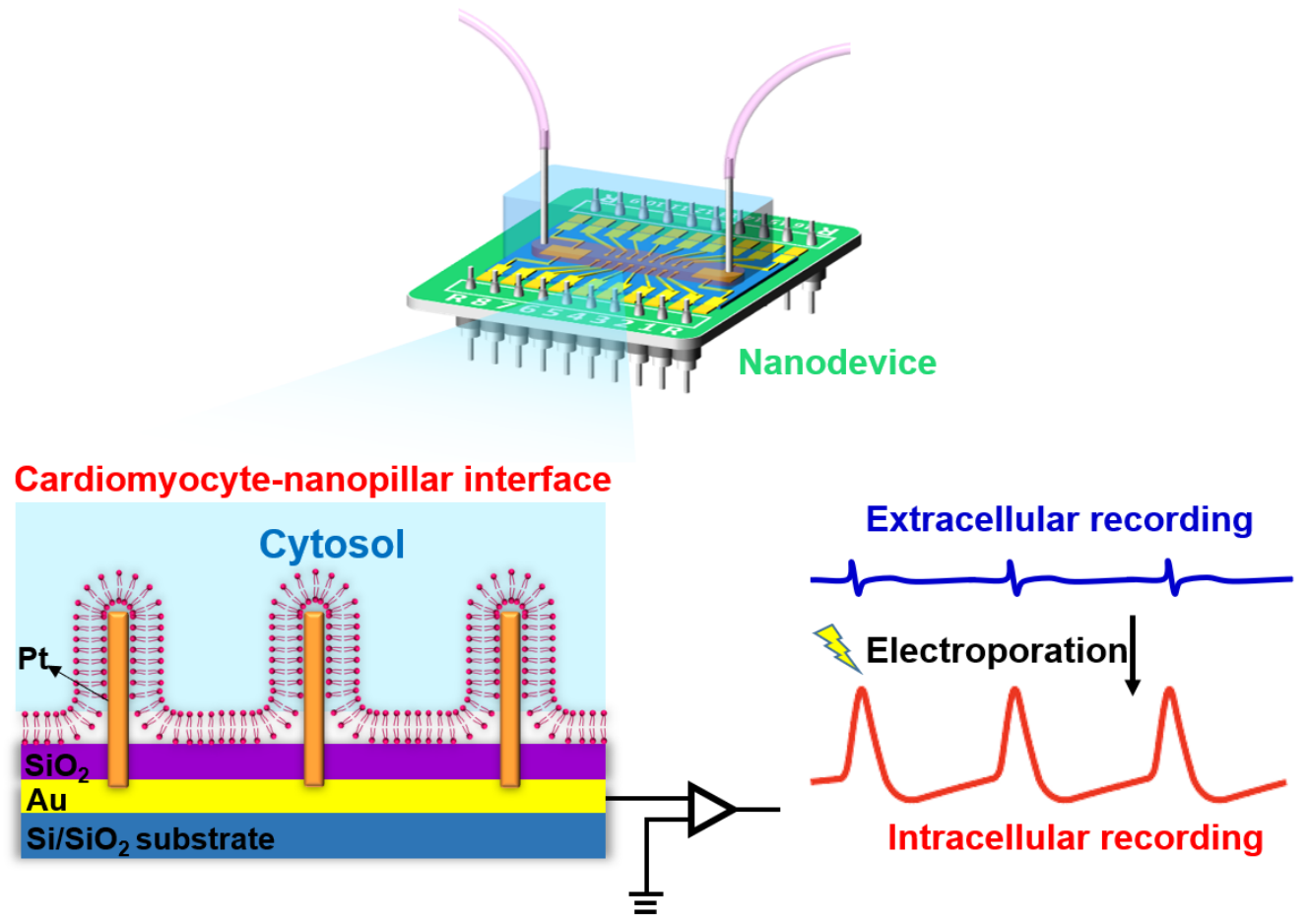

Scheme S1. Illustration of microfluidic chip with integrated nanopillar microelectrode arrays. The nanopillars formed a tight junction with the membrane of cultured HL-1 cells, enabling extracellular recordings. The nanopillars penetrated the cell membrane after electroporation, enabling intracellular recordings. 


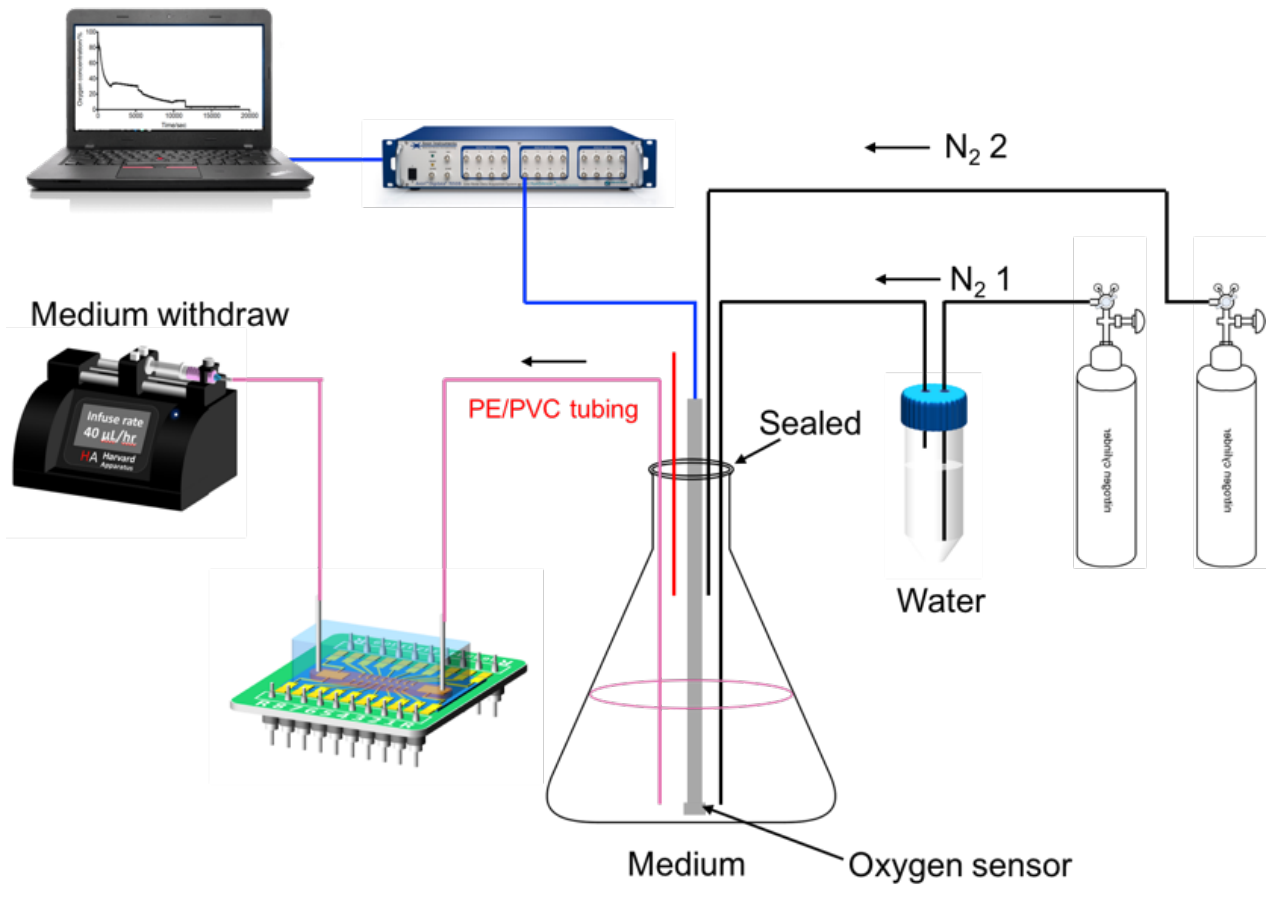

Scheme S2. Strategy to generate hypoxic medium flow. 

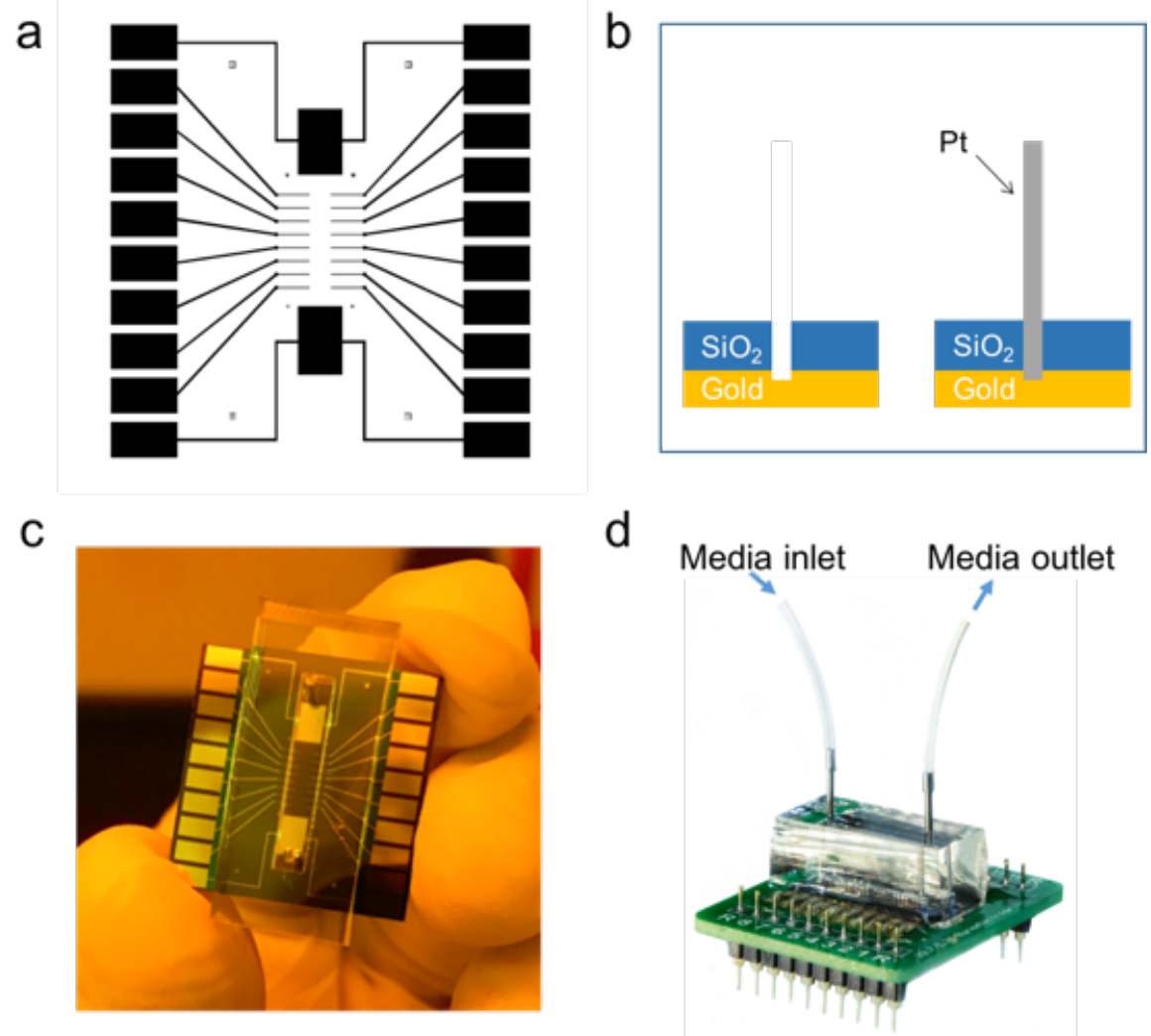

Figure S1. Design of MEA devices. (a) Mask design for photolithography. The devices consisted of 16 channels for both intracellular and extracellular signals. (b) Schematic illustrations of the nanopillar fabrication processes. (c) Optical image of the MEA device integrated with a microfluidic channel. (d) Optical image of the fully assembled device on printed circuit board. 

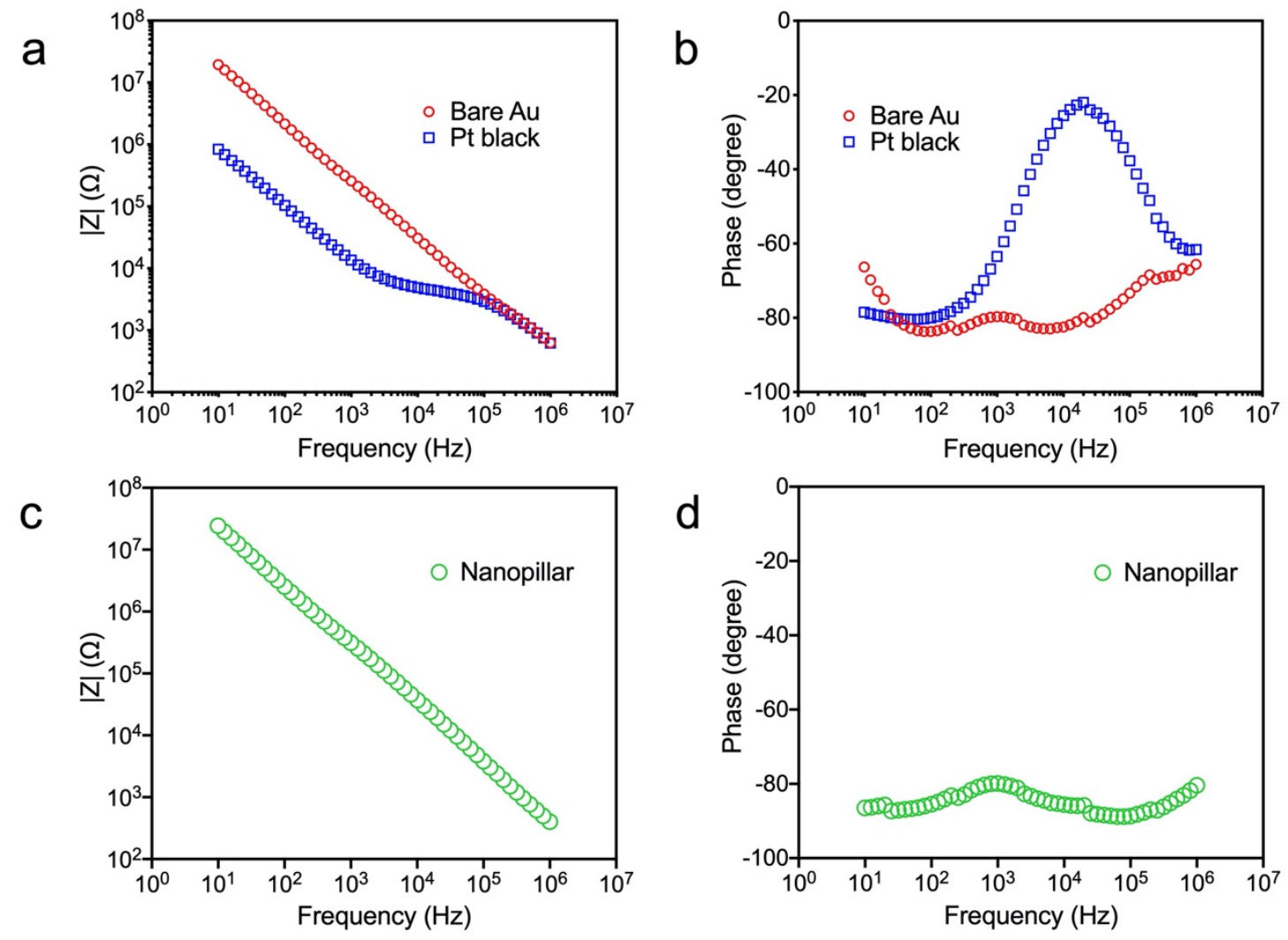

Figure S2. Representative electrical impedance spectra of (a,b) planar and (c,d) nanopillar bioelectronic devices. 


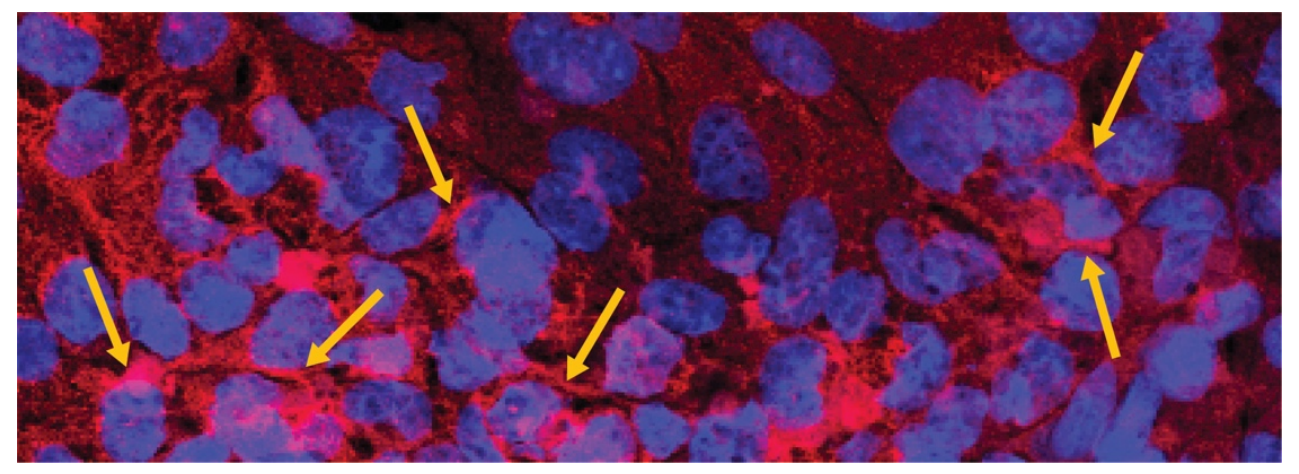

Figure S3. Gap junction localization. Immunostaining of the HL-1 cell monolayer cultured in PDMS channel at 4 DIV showing connexin-43 gap junction proteins (red) and nuclei (blue, DAPI). Yellow arrows highlight connexin-43 proteins localized at cell-cell junctions. 

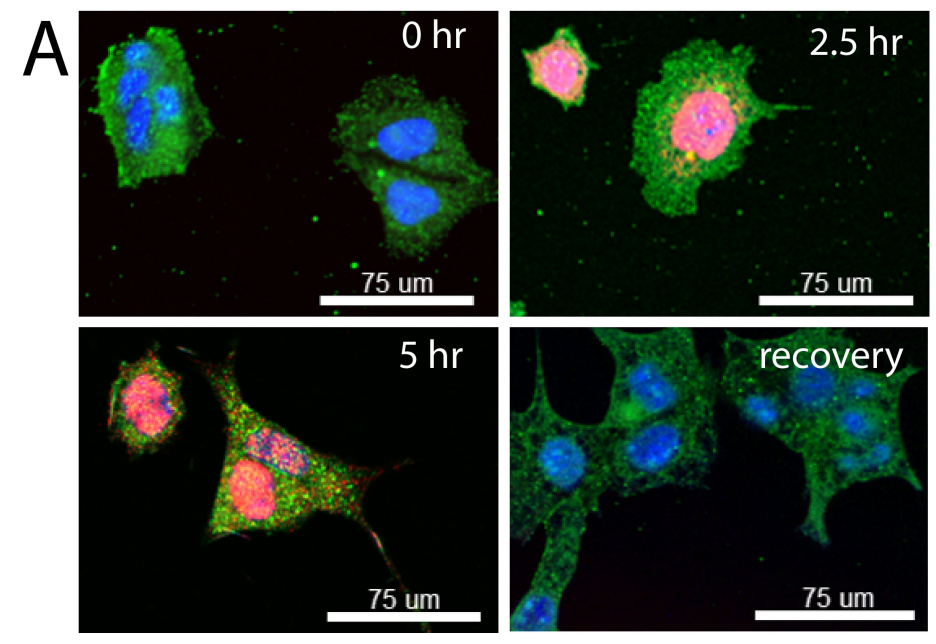

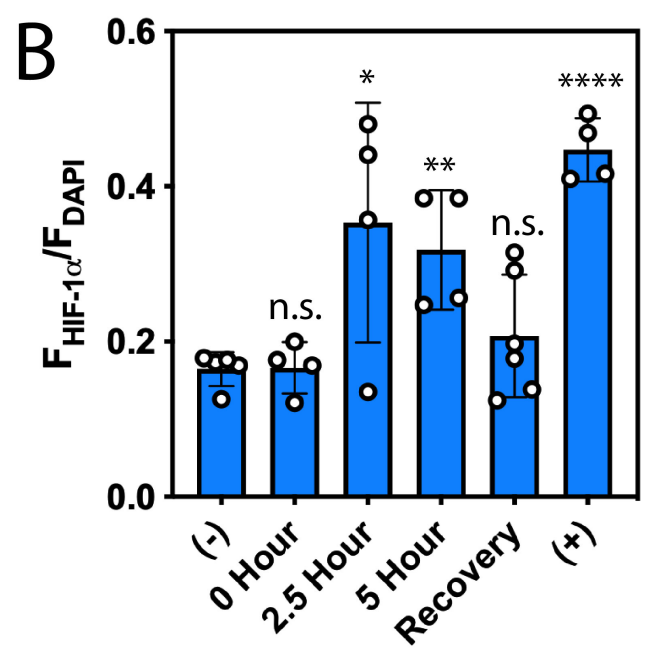

Figure S4. HIF-1 $\alpha$ validation of heart-on-a-chip. (a) Fluorescence immunostaining for HIF-1 $\alpha$ (red), DAPI (blue), and $\alpha$-actinin (green). $0 \mathrm{hr}$ time point represents normoxia; 2.5 and $5 \mathrm{hr}$ time points represent hypoxia $\left(1 \% \mathrm{O}_{2}\right)$; recovery time point was recorded 90 minutes after re-introducing normoxic media. (b) Quantification of nuclear HIF-1 $\alpha$ expression corresponding to time points shown in panel a. Negative (-) and positive (+) controls represent HL-1 cells cultured in static conditions under normoxia or in a hypoxic chamber $\left(1 \% \mathrm{O}_{2}\right)$ for $16 \mathrm{~h}$, respectively. Values are expressed as mean $\pm \mathrm{s} . \mathrm{d} . \quad * \mathrm{P}<0.05$, $* * \mathrm{P}<0.01, \mathrm{P}<0.0001$ vs. (-) by unpaired two-tailed t test. 

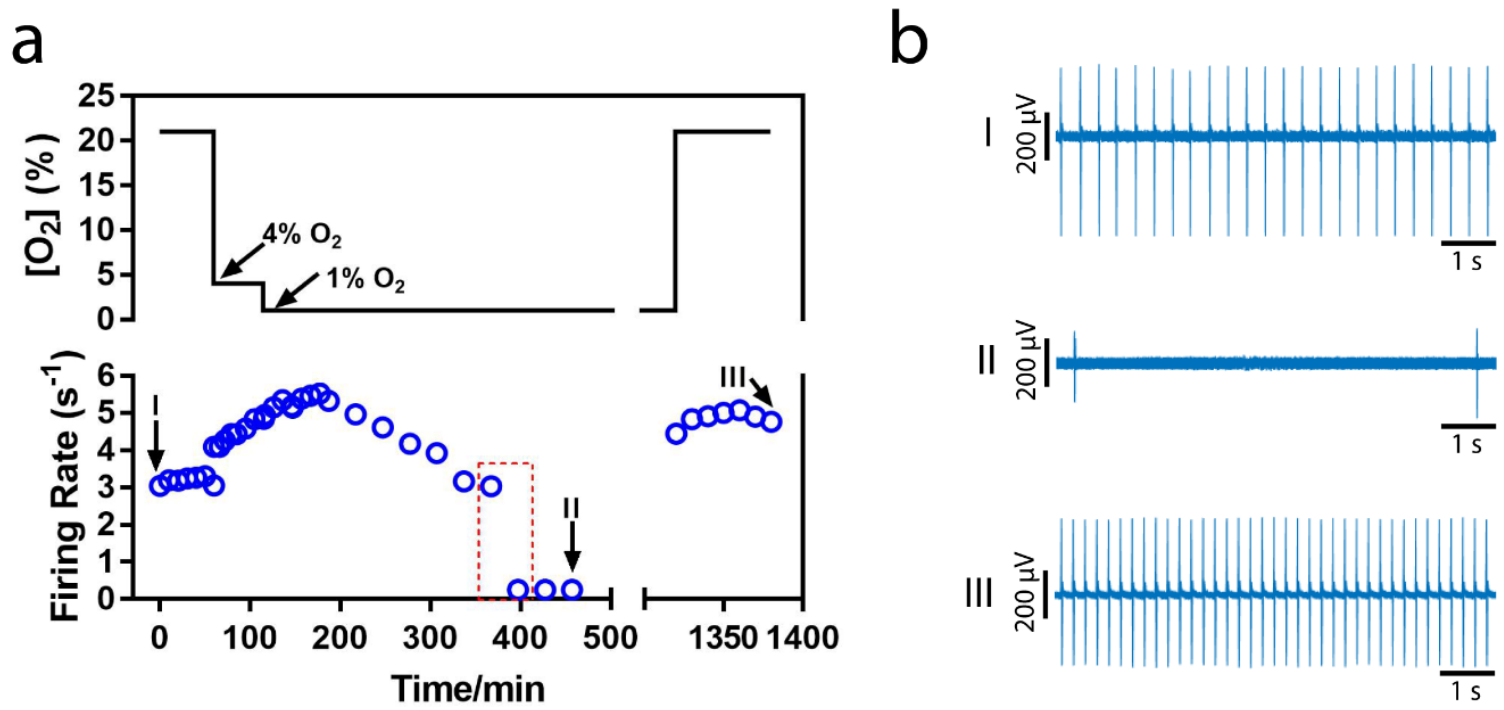

Figure S5. Extracellular bioelectronic readouts before, during and after hypoxia. (a) (top) Scheme of media delivery protocol with distinct regions of normoxia, hypoxia and recovery and (bottom) HL-1 firing rate. The red dotted box highlights the transition from rhythmic beating to arrhythmia. (b) Representative signals from a single device recorded during (I) normoxia, (II) hypoxia, upon onset of arrhythmia, and (III) recovery. These traces correspond to the points noted in panel a. 


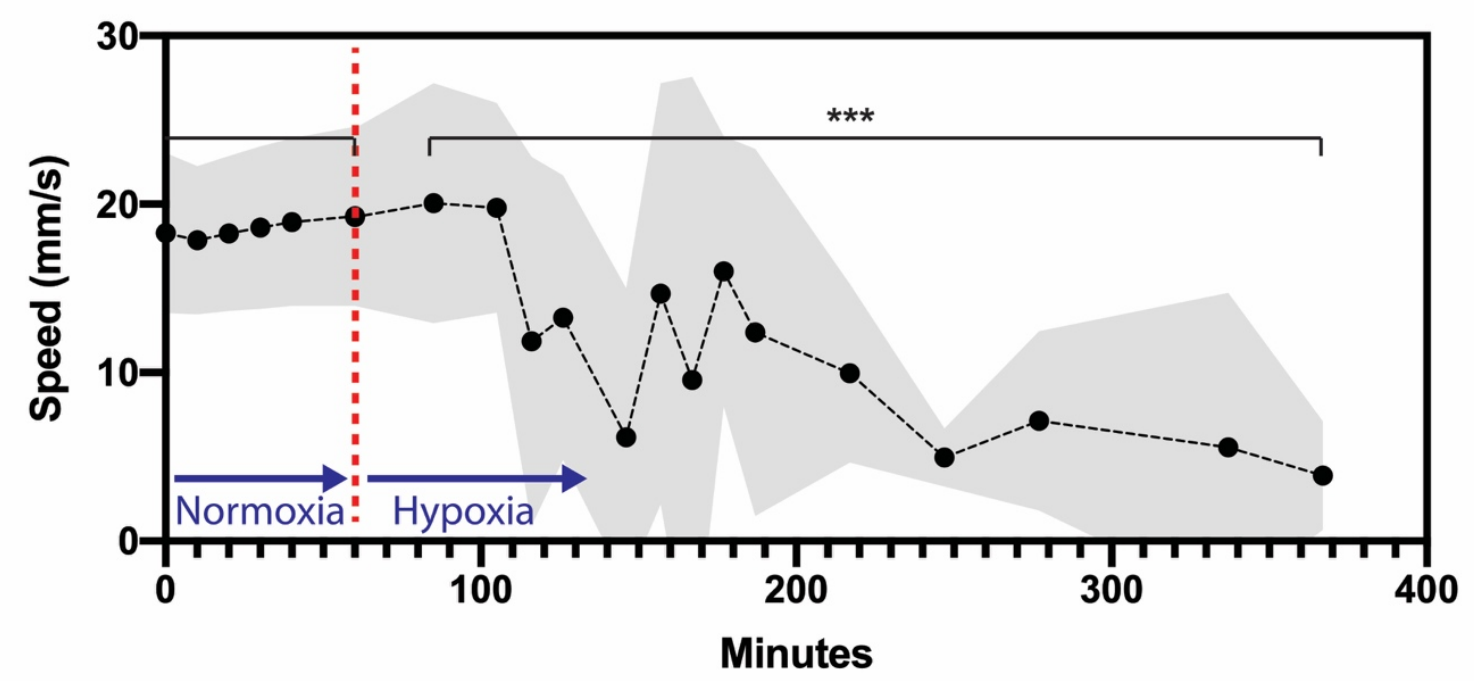

Figure S6. Summary of wavefront propagation speeds derived from isochronal maps. The medium was switched from normoxic to hypoxic at $60 \mathrm{~min}$, highlighted by the red dotted line. An isochronal map and $\mathrm{N}=105$ corresponding velocity vectors were calculated for each time point. Each point on the graph represents the average of the velocity vectors, and the grey bands represent standard deviation. ${ }^{* * *} \mathrm{P}<0.001$, Welch's t-test. 


\section{References}

1. Maoz, B. M.; Herland, A.; Henry, O. Y. F.; Leineweber, W. D.; Yadid, M.; Doyle, J.; Mannix, R.; Kujala, V. J.; FitzGerald, E. A.; Parker, K. K.; Ingber, D. E. Lab Chip 2017, 17, (13), 2294-2302.

2. Xie, C.; Lin, Z.; Hanson, L.; Cui, Y.; Cui, B. Nature nanotechnology 2012, 7, (3), 18590 .

3. Cohen-Karni, T.; Timko, B. P.; Weiss, L. E.; Lieber, C. M. Proc. Natl. Acad. Sci. U. S. A. 2009, 106, (18), 7309-13.

4. Timko, B. P.; Cohen-Karni, T.; Yu, G.; Qing, Q.; Tian, B.; Lieber, C. M. Nano Lett. 2009, 9, (2), 914-8.

5. Kim, S. B.; Bae, H.; Cha, J. M.; Moon, S. J.; Dokmeci, M. R.; Cropek, D. M.; Khademhosseini, A. Lab on a chip 2011, 11, (10), 1801-7. 